Published in final edited form as:

J Phys Chem Lett. 2020 February 06; 11(3): 832-837. doi:10.1021/acs.jpclett.9b03706.

\title{
Unperturbed Detection of the Dynamic Structure in the Hydrophobic Core of Trp-Cage via Two-Dimensional Infrared Spectroscopy
}

\author{
Farzaneh Chalyavi, Andrew J. Schmitz, Matthew J. Tucker \\ University of Nevada, Reno, Reno, Nevada
}

\begin{abstract}
The tyrosine ring mode is an intrinsic non-perturbing site-specific infrared reporter for conformational dynamics within protein systems. This transition is influenced by direct and indirect interactions associated with the electron-donating ability and the hydrophobicity of the surrounding molecules. Utilizing an intrinsic tyrosine moiety, two-dimensional infrared spectra of Trp-cage, often called the "hydrogen atom" of protein folding, were measured in the folded and denatured states to uncover the dynamics of the hydrophobic core. The vibrational lifetimes and the correlation decays of the tyrosine ring mode showed significant changes upon both temperature and chemical denaturation of the Trp-cage miniprotein, indicating important structural features of the hydrophobic core and its dynamics. The observed Trp6-Tyr3 interactions are in good agreement with the prior studies of the folded state, but they reach beyond the static structure. These stacking interactions and orientations fluctuate on the picosecond time scale as measured through the spectral dephasing within a dehydrated environment.
\end{abstract}

\section{Graphical Abstract}

Corresponding Author: mtucker@unr.edu. ASSOCIATED CONTENT

Supporting Information

The Supporting Information is available free of charge at https://pubs.acs.org/doi/10.1021/acs.jpclett.9b03706.

Vibrational lifetime decay of Trp-cage in folded and chemically denatured states, correlation decay of tyrosine in acetonitrile, vibrational lifetime decay and correlation decay of Trp-cage in temperature denaturation, and correlation decay of tyrosine in DMSO and $\mathrm{D}_{2} \mathrm{O}(\mathrm{PDF})$

The authors declare no competing financial interest. 


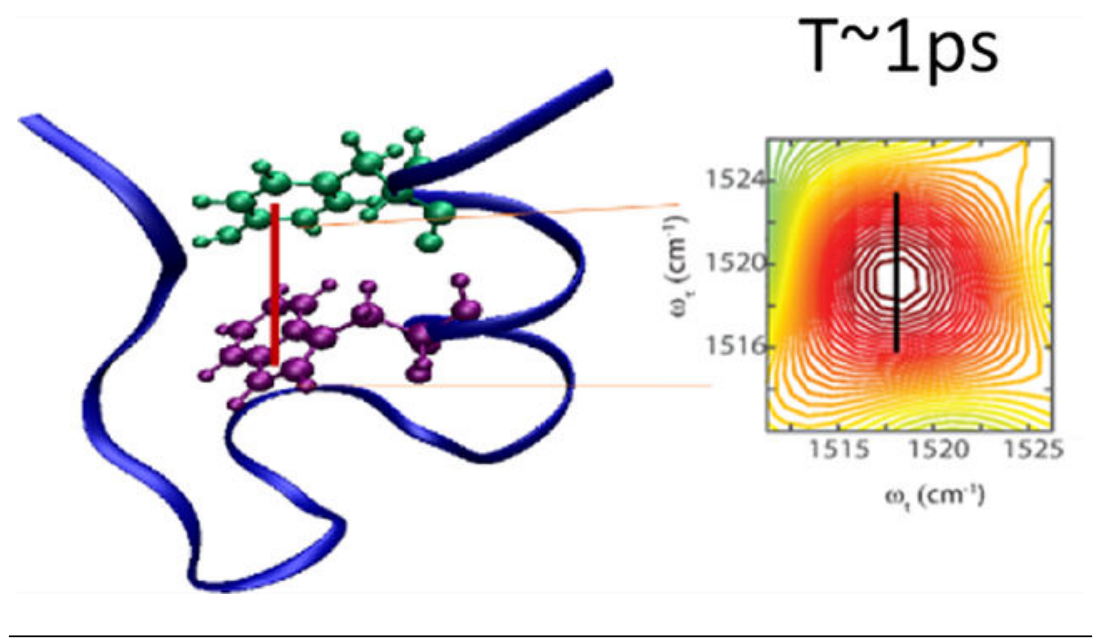

Trp-cage miniprotein, often called the hydrogen atom of folding, is one of the smallest peptide models that undergoes spontaneous fast folding ( $\sim \mu$ s) to adopt a well-defined 3D structure, and it is among the smallest and fastest foldable proteins. ${ }^{1-8}$ The small size and relatively fast folding rates of this protein allow for the development of accurate protein folding models and structure prediction algorithms, used in the determination of thermodynamic and folding properties of larger globular proteins. This miniprotein folds into a stable structure with well-defined secondary and tertiary elements. The secondary structure of the folded protein includes a hydrophobic core formed around a central tryptophan (Trp) residue. ${ }^{9,10}$ The protein tertiary structural motif includes an N-terminal ahelix, followed by a short 310 helix and a C-terminal PolyproIine II (PPII) helix. The hydrophobic core of the Trp-cage contains seven residues: Tyr3, Trp6, Leu7, Gly11, Pro12, Pro18, and Pro19. ${ }^{11-23}$ The strong and compact hydrophobic core has been of great interest for theoretical and experimental studies. ${ }^{4,6-8,24-27}$ In addition, Hagen and co-workers have investigated the folding mechanism utilizing laser temperature jump (T-jump) to reveal a two-state folding process, which has been supported by several thermodynamic studies using differential scanning calorimetry and circular dichroism spectroscopy. ${ }^{14-16,28,29}$ Other studies ${ }^{7,13,30}$ suggest the presence of a molten globule-like stable intermediate as well as an $a$-helical structure of the protein by utilizing fluorescence correlation spectroscopy and UVresonance Raman spectroscopy. Hydrophobic contacts were originally found in the protein by NMR pulse-label-experiments. NMR and IR T-jump experiments also have suggested the presence of possible intermediates in the folding mechanism and the existence of the hydrophobic/native contacts in the unfolded state. ${ }^{5,6,8,9,16,31-38}$

For this reason, several molecular dynamics (MD) simulations have attempted to predict the folding mechanism both in implicit and explicit solvent models. These studies focused on the folding pathway, various intermediate states, secondary structures, and presence of native contacts. $3,13,25,33$ The MD simulations show the critical influence of Trp6 conformations on the Trp-cage folding process. ${ }^{10} \mathrm{Gai}$ and co-workers examined the folding process of Trpcage with more detail utilizing T-jump to acquire a sequential folding mechanism. According to this study, the 310 -helix unfolds first, followed by the $a$-helix, which is required for cage formation ultimately leading to the global unfolding of the miniprotein. ${ }^{34}$ 
It is very difficult to study the key native contacts of the folded state because of limitations in side-chain probes, i.e., possible perturbation to the region of interest. Intrinsic amino acid side-chain probes can directly monitor the active site responsible for biological processes. 30,39 Understanding the dynamic structures within proteins will aid in the development of therapeutics and drug design. ${ }^{40,41}$ Typically, the amides of backbone carbonyls have been utilized to detect the presence of different secondary structures. However, side-chain probes can capture key contacts necessary to native folds and intermediate states by uncovering the site-specific interactions in the local environment. Previously, native side chains utilized as infrared probes include arginine, histidine, aspartic acid and glutamic acid carboxyl group, aromatic rings, and ester carbonyl ${ }^{42-44}$. For example, it has been shown that arginine is important for the detection of salt bridge formation. ${ }^{44}$ Histidine side chains reported on the $\mathrm{pH}$ within the M2 channel and the start of the viral replication process. ${ }^{42}$ By monitoring these types of changes around side-chain probes, detailed information about variations in the local structure can be captured. In particular, studies of the intrinsic behavior of the hydrophobic core region are difficult because of low solubility issues and the sensitivity of the core region to structural perturbations. . $^{94,45}$ To observe these important side-chain interactions within proteins requires an IR reporter that is both sensitive to the forces at work within a hydrophobic core, i.e., hydrophobicity and electron repulsion often found in conjugated systems. The tyrosine ring mode has been developed and characterized as a sitespecific IR probe that is extremely sensitive to these properties. ${ }^{46,47}$ For example, the infrared bandwidth of this mode has been shown to change because of the electron-donating ability of the solvent combined with the hydrophobicity of the solvent. In fact, a linear correlation was uncovered that relates variations in the bandwidth of the tyrosine ring mode with changes in the solvent electrostatic field. Interestingly, the Trp-cage miniprotein contains a native tyrosine residue directly involved in the hydrophobic core formation. ${ }^{46}$

As mentioned above, a linear correlation of the IR bandwidth was determined as a function of the electric field strength by observing the tyrosine ring mode ${ }^{46}$ This correlation was utilized to detect the electric field strength, i.e., local environment, of the hydrophobic core region found in the Trp-cage. An observable change in the electric field was measured between folded and denatured states. From the experimental bandwidth (Figure 1), the interior of the hydrophobic core within Trp-cage was suggested to have a similar local electric field as induced by the solvent acetonitrile $(\sim 20.52 \mathrm{MV} / \mathrm{cm})$ for the native folded state. Upon chemical denaturation, the bandwidth of the tyrosine ring mode in the core region of the peptide recovered the total bandwidth observed for tyrosine in water. Although the vibrational bandwidth is sensitive to denaturation, the sources of these bandwidth changes remain in question. Also, the variations in the bandwidth are moderately small. ${ }^{46}$ Thus, in order to have more precise measurements of the dynamics and identify the likely interactions responsible, two-dimensional infrared (2D IR) spectroscopy was invoked, and the sources of the possible interactions leading to spectral dephasing were uncovered with the help of prior structural information. ${ }^{1,38}$ Moreover, significant differences in the vibrational lifetimes of the tyrosine ring mode within the Trp-cage were observed via 2D IR spectroscopy.

The FTIR spectra of Trp-cage miniprotein in water and the chemically denatured ( $\mathrm{GdnHCl})$ state of Trp-cage in solution at room temperature $\left(\sim 25^{\circ} \mathrm{C}\right)$ were measured, as shown in 
Figure 2. Similar to prior results, ${ }^{46}$ the vibrational bandwidth of the tyrosine ring mode in the folded state $\left(\sim 6.2 \mathrm{~cm}^{-1}\right)$ was $\sim 26 \%$ larger than that of the chemically denatured state $(\sim 2$ $\mathrm{M} \mathrm{GdnHCl}) .{ }^{48}$ Additionally, the intensity of the IR transition, i.e., the absorption cross section, decreases by half upon denaturation, likely because of fast homogeneous dephasing as observed in different solvents. The major contributions to the linear IR bandwidth are due to the population relaxation, i.e., the vibrational lifetime, and frequency-frequency correlation decay resulting from homogeneous and inhomogeneous dephasing terms. Thus, 2D IR measurements have been performed to investigate these major contributions.

The waiting time dependence, $T=0-2100 \mathrm{fs}$ with $150 \mathrm{fs}$ steps, of the 2D IR spectra was obtained for both the folded and chemically denatured states of the Trp-cage miniprotein. The 2D IR spectrum of the Trp-cage in the folded state shows a $0 \rightarrow 1$ transition, located at $\omega_{\mathrm{t}}=\omega_{\tau}=1518 \mathrm{~cm}^{-1}$, with the $1 \rightarrow 2$ transition anharmonically shifted by $11.7 \pm 0.9 \mathrm{~cm}^{-1}$. Similarly, the 2D IR spectra of Trp-cage in the denatured state exhibited the $0 \rightarrow 1$ transition is located at $\omega_{\mathrm{t}}=\omega_{\tau}=1517 \mathrm{~cm}^{-1}$ with a slightly larger anharmonicity of $14.5 \pm 1.8$ $\mathrm{cm}^{-1}$. Considering prior studies, ${ }^{46}$ these values are somewhat expected given the 2D IR of the tyrosine amino acid in $\mathrm{D}_{2} \mathrm{O}$ and organic solvent, i.e., DMSO. As a result of the population relaxation $\left(T_{10}\right)$, the positive signals decay during the waiting time, $T$, with a time constant equal to $4.6 \pm 0.4$ ps and $2.1 \pm 0.2 \mathrm{ps}$ in folded and denatured states, respectively (Figure S1). Assuming single-exponential decays of the diagonal signals, the relaxation time is equal to the time when only $37 \%$ of the signal remains. The lifetime decays of the tyrosine moiety in the denatured Trp-cage and the tyrosine amino acid in water $(\sim 2.2 \pm 0.2 \mathrm{ps})^{46}$ are in agreement, suggesting that the ring mode in chemically denatured Trp-cage is in a similar environment, i.e., solvent exposed. However, a slower lifetime decay is observed for the folded state of the miniprotein. Zanni and co-workers have shown that the degree of solvation can significantly alter the vibrational lifetime. ${ }^{49}$ In addition, the amount of mobile water in the Trp-cage has been suggested by NMR and MD simulations to be composed of different degrees of hydration between the folded and disordered states. ${ }^{35}$ Therefore, it is not surprising that the strong hydrophobic core would have a significantly lesser degree of solvation.

In addition to the signal intensity changes, both diagonal peaks are tilted and elongated along the diagonal at the earliest times as a result of inhomogeneous broadening. By observing the positive diagonal signals of the 2D IR spectra at $T=150$ and 1200 fs (Figure 3 ), the inverse of the center line slope (CLS) changes as the peaks become more circular, giving evidence of the loss of frequency correlation. It is clear from the changes in the spectral shape and the slopes that the dephasing is faster in the hydrophobic core than in the bulk water. For a more detailed analysis, the correlation decays have been determined by the CLS method. ${ }^{50,51}$ It should be noted that the initial magnitude of the inverse slope for both cases is somewhat smaller than other oscillators, as observed before for the tyrosine ring mode,${ }^{46}$ suggesting that some fast motions have already caused significant spectral dephasing. Assuming a single exponential decay, the correlation decay in the folded state was determined to be $968 \pm 77 \mathrm{fs}$, while upon chemical denaturation the decay is $1.6 \times$ longer (1500 $\pm 100 \mathrm{fs}$ ) (Figure 4). The faster dephasing in the folded state is similar to that reported for tyrosine in DMSO (see Figure S5). The electric field around the tyrosine residue in the hydrophobic core was shown to be similar to the tyrosine in an acetonitrile (ACN) 
environment (Figure 1). For this reason, a direct comparison was made between the correlation decays in the folded state and tyrosine in acetonitrile. The time scale of the decay in both cases was $\sim 1.0$ ps (see Figure S2), demonstrating that electron-donating ability within ACN is quite similar to that experienced by tyrosine in the hydrophobic core. However, the source of the dephasing (i.e., from the electron repulsion to the ring) must be somewhat different within the core region of the peptide. Upon inspection of the NMR structure (PDB 1L2Y), the average value of the distance between tryptophan lone pair electrons and tyrosine in the hydrophobic core is about $6.6 \AA$. According to prior DFT computations on the ring mode, the vibrational frequency will vary significantly between distances of 2.4 and $8.3 \AA$ because of the repulsive interactions between lone pair electrons and the conjugated ring. ${ }^{46}$ Thus, these results suggest that tryptophan and tyrosine are closely stacked, and the relative orientation between the rings fluctuates within a distance range to allow the lone pair electrons on the tryptophan to affect the ring mode frequency. In addition, variations in dynamics may result from fast spontaneous fluctuations of the Tyr residue itself. Thus, the motions most likely responsible for the spectral diffusion are related to the rotation of the side chains (i.e., the rotamer wagging motions), which have angles ranging from $21^{\circ}$ to $77^{\circ}$. Overall, these stacking interactions observed in the hydrophobic core are stabilized by hydration effects but encounter a delicate balance with prior mentioned repulsive interactions.

In addition to chemical denaturation, 2D IR spectra of Trp-cage were collected at a temperature of $\sim 50{ }^{\circ} \mathrm{C}$, which is above the melting temperature of the protein causing thermal denaturation. ${ }^{52}$ The lifetime and correlation decays were determined as described above. The vibrational lifetime, $1.7 \pm 0.1 \mathrm{ps}$, for the temperature denatured state of Trp-cage is 0.4 ps different from the chemically denatured state (Figure S3). This slight difference can be attributed to faster thermal relaxation at a higher temperature, which has been shown to decrease the vibrational lifetime. ${ }^{53,54}$ On the other hand, the correlation decay time, 1.2 $\pm 0.2 \mathrm{ps}$, for the temperature denatured state is halfway between the folded and unfolded state decay times (Figure S4). These results indicate that the hydrophobic core remains at least partially intact during the temperature denaturation process, further suggesting the possibility of a molten globule-like stable intermediate. Also, the miniprotein does not become fully unfolded until it is chemically denatured. Overall, these findings support the folding mechanism proposed by Gai and co-workers in which the hydrophobic core is the major stabilizing force, with the $a$-helix also playing a role in stabilization, during the folding process. 9,34

Using 2D IR spectroscopy, the structure and dynamics of the hydrophobic core region of Trp-cage miniprotein were investigated utilizing the unperturbative native vibrational reporter, the ring mode of tyrosine within the core. Considering previous studies of the linear correlation between the IR bandwidth and electric field, it was expected that the vibrational dynamics, resulting from structural movements and solvent effects, would be different between the folded and the denatured state. Now, the origin of these differences has been identified by observing the vibrational lifetime and correlation decays via 2D IR spectroscopy. The slower vibrational lifetime of the folded state shows the hydrophobic core is dehydrated. More importantly, the stacking effect between Trp and Tyr within the core is responsible for the overall stability but retains a fluidity in its structural dynamics to result in 
the faster dephasing in the folded state. These spectral features uncovered in this study can be utilized in computational studies for investigating the hydration state in the hydrophobic core as well as relative orientation and distance between the ring and the lone pair to see the effect on the vibration frequency. Our results also further support the need for an additional stabilization factor of the core region, possibly from the $a$-helix. Although the Trp6-Tyr3 interactions uncovered in these studies are expected in the folded state, our results reach beyond the static structure. Overall, these stacking interactions and/or orientations between Trp and Tyr fluctuate over distances that vary by $5 \AA$ on the picosecond time scale, within a dehydrated environment.

\section{EXPERIMENTAL METHODS}

Trp-cage was synthesized by a standard Fmoc solid-phase protocol on the aapptec Focus XC peptide synthesizer. The peptide was purified by reverse-phase HPLC with a Vydac C18 column and characterized by a Bruker MALDI-TOF mass spectrometry instrument. In our studies, the Trp-cage concentration was $\sim 0.01 \mathrm{mg} / \mathrm{mL}$. For chemical denaturation studies 20 $\mathrm{mM}$ sodium phosphate buffer at $\mathrm{pH} 7$ with $2 \mathrm{M}$ guanidine hydrochloride was added to the peptide.

The IR spectra were collected using a ThermoNicolet 6700 FTIR spectrometer, equipped with a liquid nitrogen cooled mercury cadmium telluride detector, at $1 \mathrm{~cm}^{-1}$ using a homemade two-compartment $\mathrm{CaF}_{2}$ sample cell with a $56 \mu \mathrm{m}$ Teflon spacer. In this way, measurements of the reference and the sample were performed under the same experimental conditions. An automated translation stage moves the sample cell between the reference and the sample side collecting a single beam spectrum for each side.

\section{D IR Methods}

Heterodyned spectral interferometry was utilized for obtaining the spectra. Fourier transform-limited $80 \mathrm{fs}$ pulses with a central wavelength of $6591 \mathrm{~nm}$ were employed in the 2D IR experiments. Three $\sim 1 \mu \mathrm{J}$ laser pulses with wave vectors $k_{1}, k_{2}$, and $k_{3}$ were incident to the sample generating a signal in the direction $k_{\mathrm{s}}=-k_{1}+k_{2}+k_{3}$ with the ordering 123 (rephasing) and 213 (nonrephasing). To obtain absorptive spectra, the rephasing and nonrephasing $2 \mathrm{D}$ frequency spectra were properly phased and combined. To observe any changes in the spectral characteristics, the waiting time, $T$, between the second and third pulse was varied from 0 to $2 \mathrm{ps}$. After appropriate Fourier transforms along the coherence, $\tau$, and detection, $\mathrm{t}$, axes, the 2D IR spectra were plotted as $\omega_{\tau}$ versus $\omega_{\mathrm{t}}{ }^{55}$

\section{Supplementary Material}

Refer to Web version on PubMed Central for supplementary material.

\section{ACKNOWLEDGMENTS}

The research was supported by an NIH (R15GM1224597) grant to M.J.T. 


\section{REFERENCES}

(1). Neidigh JW; Fesinmeyer RM; Andersen NH Designing a 20-Residue Protein. Nat. Struct. Biol 2002, 9 (6), 425-430. [PubMed: 11979279]

(2). Qiu L; Pabit SA; Roitberg AE; Hagen SJ Smaller and Faster: The 20-Residue Trp-Cage Protein Folds in 4 Ms. J. Am. Chem. Soc 2002, 124 (44), 12952-12953. [PubMed: 12405814]

(3). Pitera JW; Swope W Understanding Folding and Design: Replica-Exchange Simulations of TrpCage Miniproteins. Proc. Natl. Acad. Sci. U. S. A 2003, 100 (13), 7587-7592. [PubMed: 12808142]

(4). Simmerling C; Strockbine B; Roitberg AE All-Atom Structure Prediction and Folding Simulations of a Stable Protein. J. Am. Chem. Soc 2002, 124 (38), 11258-11259. [PubMed: 12236726]

(5). Snow CD; Zagrovic B; Pande VS The Trp Cage: Folding Kinetics and Unfolded State Topology via Molecular Dynamics Simulations. J. Am. Chem. Soc 2002, 124 (49), 14548-14549. [PubMed: 12465960]

(6). Meuzelaar H; Marino KA; Huerta-Viga A; Panman MR; Smeenk LEJ; Kettelarij AJ; Van Maarseveen JH; Timmerman P; Bolhuis PG; Woutersen S Folding Dynamics of the Trp-Cage Miniprotein: Evidence for a Native-like Intermediate from Combined Time-Resolved Vibrational Spectroscopy and Molecular Dynamics Simulations. J. Phys. Chem. B 2013, 117 (39), 1149011501. [PubMed: 24050152]

(7). Neuweiler H; Doose S; Sauer M A Microscopic View of Miniprotein Folding: Enhanced Folding Efficiency through Formation of an Intermediate. Proc. Natl. Acad. Sci. U. S. A 2005, 102 (46), 16650-16655. [PubMed: 16269542]

(8). Barua B; Lin JC; Williams VD; Kummler P; Neidigh JW; Andersen NH The Trp-Cage: Optimizing the Stability of a Globular Miniprotein. Protein Eng., Des. Sel 2008, 21 (3), 171-185. [PubMed: 18203802]

(9). Bunagan MR; Yang X; Saven JG; Gai F Ultrafast Folding of a Computationally Designed TrpCage Mutant: Trp 2-Cage. J. Phys. Chem. B 2006, 110 (8), 3759-3763. [PubMed: 16494434]

(10). Kannan S; Zacharias M Role of Tryptophan Side Chain Dynamics on the Trp-Cage Mini-Protein Folding Studied by Molecular Dynamics Simulations. PLoS One 2014, 9 (2), e88383. [PubMed: 24563686]

(11). Zheng W; Gallicchio E; Deng N; Andrec M; Levy RM Kinetic Network Study of the Diversity and Temperature Dependence of Trp-Cage Folding Pathways: Combining Transition Path Theory with Stochastic Simulations. J. Phys. Chem. B 2011, 115 (6), 1512-1523. [PubMed: 21254767]

(12). Bruce NJ; Bryce RA Ab Initio Protein Folding Using a Cooperative Swarm of Molecular Dynamics Trajectories. J. Chem. Theory Comput 2010, 6 (7), 1925-1930. [PubMed: 26615921]

(13). Paschek D; Nymeyer H; García AE Replica Exchange Simulation of Reversible Folding/ Unfolding of the Trp-Cage Miniprotein in Explicit Solvent: On the Structure and Possible Role of Internal Water. J. Struct. Biol 2007, 157 (3), 524-533. [PubMed: 17293125]

(14). Seshasayee ASN High-Temperature Unfolding of a Trp-Cage Mini-Protein: A Molecular Dynamics Simulation Study. Theor. Biol. Med. Modell 2005, 2, 1-5.

(15). Zhou CY; Jiang F; Wu YD Folding Thermodynamics and Mechanism of Five Trp-Cage Variants from Replica-Exchange MD Simulations with RSFF2 Force Field. J. Chem. Theory Comput 2015, 11 (11), 5473-5480. [PubMed: 26574335]

(16). Paschek D; Hempel S; García AE Computing the Stability Diagram of the Trp-Cage Miniprotein. Proc. Natl. Acad. Sci. U. S. A 2008, 105 (46), 17754-17759. [PubMed: 19004791]

(17). Lee MS; Olson MA Protein Folding Simulations Combining Self-Guided Langevin Dynamics and Temperature-Based Replica Exchange. J. Chem. Theory Comput 2010, 6 (8), 2477-2487. [PubMed: 26613500]

(18). Cerny J; Vondrasek J; Hobza P Loss of Dispersion Energy Changes the Stability and Folding/ Unfolding Equilibrium of the Trp-Cage Protein. J. Phys. Chem. B 2009, 113, 5657-5660. [PubMed: 19444987]

(19). Marinelli F; Pietrucci F; Laio A; Piana S A Kinetic Model of Trp-Cage Folding from Multiple Biased Molecular Dynamics Simulations. PLoS Comput. Biol. 2009, 5 (8), e1000452. [PubMed: 19662155] 
(20). Piana S; Laio A A Bias-Exchange Approach to Protein Folding. J. Phys. Chem. B 2007, 111 (17), 4553-4559. [PubMed: 17419610]

(21). Chen J; Im W; Brooks CL Balancing Solvation and Intramolecular Interactions: Toward a Consistent Generalized Born Force Field. J. Am. Chem. Soc 2006, 128 (11), 3728-3736. [PubMed: 16536547]

(22). Ding F; Buldyrev SV; Dokholyan NV Folding Trp-Cage to NMR Resolution Native Structure Using a Coarse-Grained Protein Model. Biophys. J 2005, 88 (1), 147-155. [PubMed: 15533926]

(23). Yang L; Grubb MP; Gao YQ Application of the Accelerated Molecular Dynamics Simulations to the Folding of a Small Protein. J. Chem. Phys 2007, 126 (12), 125102. [PubMed: 17411164]

(24). Lee IH; Kim SY Dynamic Folding Pathway Models of the Trp-Cage Protein. BioMed Res. Int 2013, 2013, 973867. [PubMed: 23865078]

(25). Zhou R Free Energy Landscape of Protein Folding in Water: Explicit vs. Implicit Solvent. Proteins: Struct., Funct., Genet 2003, 53 (2), 148-161. [PubMed: 14517967]

(26). Hudáky P; Stráner P; Farkas V; Váradi G; Tóth G; Perczel A Cooperation between a Salt Bridge and the Hydrophobic Core Triggers Fold Stabilization in a Trp-Cage Miniprotein. Biochemistry 2008, 47 (3), 1007-1016. [PubMed: 18161949]

(27). Linhananta A; Boer J; MacKay I The Equilibrium Properties and Folding Kinetics of an AllAtom G o- Model of the Trp-Cage. J. Chem. Phys 2005, 122 (11), 114901. [PubMed: 15836251]

(28). Streicher WW; Makhatadze GI Unfolding Thermodynamics of Trp-Cage, a 20 Residue Miniprotein, Studied by Differential Scanning Calorimetry and Circular Dichroism Spectroscopy. Biochemistry 2007, 46 (10), 2876-2880. [PubMed: 17295518]

(29). Hałabis A; Zmudzińska W; Liwo A; Ołdziej S Conformational Dynamics of the Trp-Cage Miniprotein at Its Folding Temperature. J. Phys. Chem. B 2012, 116 (23), 6898-6907. [PubMed: 22497240]

(30). Lin C-W; Mensa B; Barniol-Xicota M; DeGrado WF; Gai F Activation PH and Gating Dynamics of Influenza A M2 Proton Channel Revealed by Single-Molecule Spectroscopy. Angew. Chem., Int. Ed 2017, 56 (19), 5283-5287.

(31). English CA; García AE Folding and Unfolding Thermodynamics of the TC10b Trp-Cage Miniprotein. Phys. Chem. Chem. Phys 2014, 16 (7), 2748-2757. [PubMed: 24448113]

(32). Byrne A; Williams DV; Barua B; Hagen SJ; Kier BL; Andersen NH Folding Dynamics and Pathways of the Trp-Cage Miniproteins. Biochemistry 2014, 53 (38), 6011-6021. [PubMed: 25184759]

(33). Zhou R Trp-Cage: Folding Free Energy Landscape in Explicit Water. Proc. Natl. Acad. Sci. U. S. A 2003, 100 (23), 13280-13285. [PubMed: 14581616]

(34). Culik RM; Serrano AL; Bunagan MR; Gai F Achieving Secondary Structural Resolution in Kinetic Measurements of Protein Folding: A Case Study of the Folding Mechanism of Trp-Cage. Angew. Chem., Int. Ed 2011, 50, 10884-10887.

(35). Taricska N; Bokor M; Menyhárd DK; Tompa K; Perczel A Hydration Shell Differentiates Folded and Disordered States of a Trp-Cage Miniprotein, Allowing Characterization of Structural Heterogeneity by Wide-Line NMR Measurements. Sci. Rep 2019, 9 (1), 2947. [PubMed: 30814556]

(36). Wafer LNR; Streicher WW; Makhatadze GI Thermodynamics of the Trp-Cage Miniprotein Unfolding in Urea. Proteins: Struct., Funct., Genet 2010, 78 (6), 1376-1381. [PubMed: 20112418]

(37). Ghosh K; Ozkan SB; Dill KA The Ultimate Speed Limit to Protein Folding Is Conformational Searching. J. Am. Chem. Soc 2007, 129 (39), 11920-11927. [PubMed: 17824609]

(38). Scian M; Lin JC; Le Trong I; Makhatadze GI; Stenkamp RE; Andersen NH Crystal and NMR Structures of a Trp-Cage Mini-Protein Benchmark for Computational Fold Prediction. Proc. Natl. Acad. Sci. U. S. A 2012, 109 (31), 12521-12525. [PubMed: 22802678]

(39). Kratochvil HT; Carr JK; Matulef K; Annen AW; Li H; Maj M; Ostmeyer J; Serrano AL; Raghuraman H; Moran SD; et al. Instantaneous Ion Configurations in the K+ Ion Channel Selectivity Filter Revealed by 2D IR Spectroscopy. Science (Washington, DC, U. S.) 2016, 353, 1040-1044. 
(40). Fang C; Bauman JD; Das K; Remorino A; Arnold E; Hochstrasser RM Two-Dimensional Infrared Spectra Reveal Relaxation of the Nonnucleoside Inhibitor TMC278 Complexed with HIV-1 Reverse Transcriptase. Proc. Natl. Acad. Sci. U. S. A 2008, 105 (5), 1472-1477. [PubMed: 18040050]

(41). Kuroda DG; Bauman JD; Challa JR; Patel D; Troxler T; Das K; Arnold E; Hochstrasser RM Snapshot of the Equilibrium Dynamics of a Drug Bound to HIV-1 Reverse Transcriptase. Nat. Chem 2013, 5 (3), 174-181. [PubMed: 23422558]

(42). Ghosh A; Tucker MJ; Gai F 2D IR Spectroscopy of Histidine: Probing Side-Chain Structure and Dynamics via Backbone Amide Vibrations. J. Phys. Chem. B 2014, 118 (28), 7799-7805. [PubMed: 24712671]

(43). Pazos IM; Ghosh A; Tucker MJ; Gai F Ester Carbonyl Vibration as a Sensitive Probe of Protein Local Electric Field. Angew. Chem., Int. Ed 2014, 53, 6080-6084.

(44). Ghosh A; Tucker MJ; Hochstrasser RM Identification of Arginine Residues in Peptides by 2D-IR Echo Spectroscopy. J. Phys. Chem. A 2011, 115 (34), 9731-9738. [PubMed: 21539337]

(45). Rovó P; Stráner P; Láng A; Bartha I; Huszár K; Nyitray L; Perczel A Structural Insights into the Trp-Cage Folding Intermediate Formation. Chem. - Eur. J 2013, 19 (8), 2628-2640. [PubMed: 23319425]

(46). Chalyavi F; Hogle DG; Tucker MJ Tyrosine as a Non-Perturbing Site-Specific Vibrational Reporter for Protein Dynamics. J. Phys. Chem. B 2017, 121 (26), 6380-6389. [PubMed: 28590738]

(47). Sul S; Feng Y; Le U; Tobias DJ; Ge NH Interactions of Tyrosine in Leu-Enkephalin at a Membrane-Water Interface: An Ultrafast Two-Dimensional Infrared Study Combined with Density Functional Calculations and Molecular Dynamics Simulations. J. Phys. Chem. B 2010, 114 (2), 1180-1190. [PubMed: 20017523]

(48). Heyda J; Kožíšek M; Bednárova L; Thompson G; Konvalinka J; Vondrášek J; Jungwirth P Urea and Guanidinium Induced Denaturation of a Trp-Cage Miniprotein. J. Phys. Chem. B 2011, 115 (28), 8910-8924. [PubMed: 21634379]

(49). Middleton CT; Buchanan LE; Dunkelberger EB; Zanni MT Utilizing Lifetimes to Suppress Random Coil Features in 2D IR Spectra of Peptides. J. Phys. Chem. Lett 2011, 2 (18), 23572361. [PubMed: 21966585]

(50). Kwak K; Park S; Finkelstein IJ; Fayer MD Frequency-Frequency Correlation Functions and Apodization in Two-Dimensional Infrared Vibrational Echo Spectroscopy: A New Approach. J. Chem. Phys 2007, 127 (12), 124503. [PubMed: 17902917]

(51). Kwak K; Rosenfeld DE; Fayer MD Taking Apart the Two-Dimensional Infrared Vibrational Echo Spectra: More Information and Elimination of Distortions. J. Chem. Phys 2008, 128 (20), 204505. [PubMed: 18513030]

(52). Irbäck A; Mohanty S Folding Thermodynamics of Peptides. Biophys. J 2005, 88 (3), 1560-1569. [PubMed: 15613623]

(53). Myers DJ; Urdahl RS; Cherayil BJ; Fayer MD Temperature Dependence of Vibrational Lifetimes at the Critical Density in Supercritical Mixtures. J. Chem. Phys 1997, 107 (23), 9741-9748.

(54). Tokmakoff A; Sauter B; Fayer MD Temperature-Dependent Vibrational Relaxation in Polyatomic Liquids: Picosecond Infrared Pump-Probe Experiments. J. Chem. Phys 1994, 100 (12), 90359043.

(55). Kim YS; Wang J; Hochstrasser RM Two-Dimensional Infrared Spectroscopy of the Alanine Dipeptide in Aqueous Solution. J. Phys. Chem. B 2005, 109, 7511-7521. [PubMed: 16851862] 


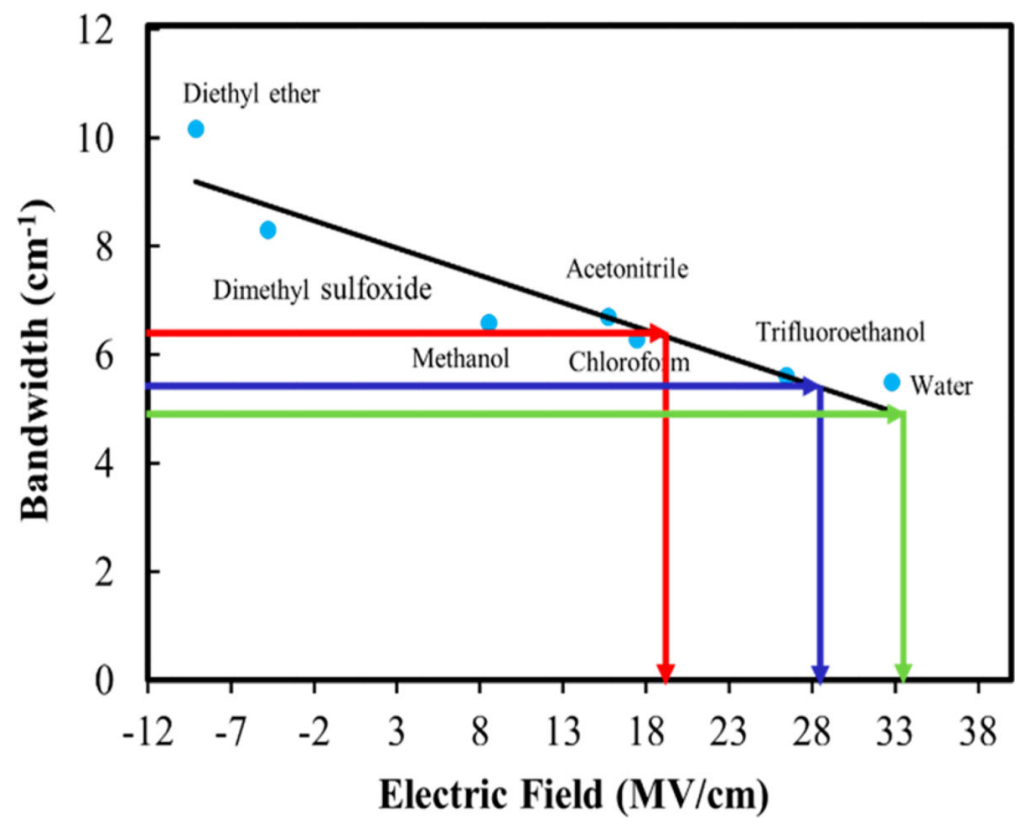

Figure 1.

Linear correlation between the linear infrared bandwidth of tyrosine transition and the electric field tested in Trp-cage miniprotein folded (red), temperature denatured (blue), and chemically denatured (green) states, adapted from ref 46. Copyright 2017 American Chemical Society. 

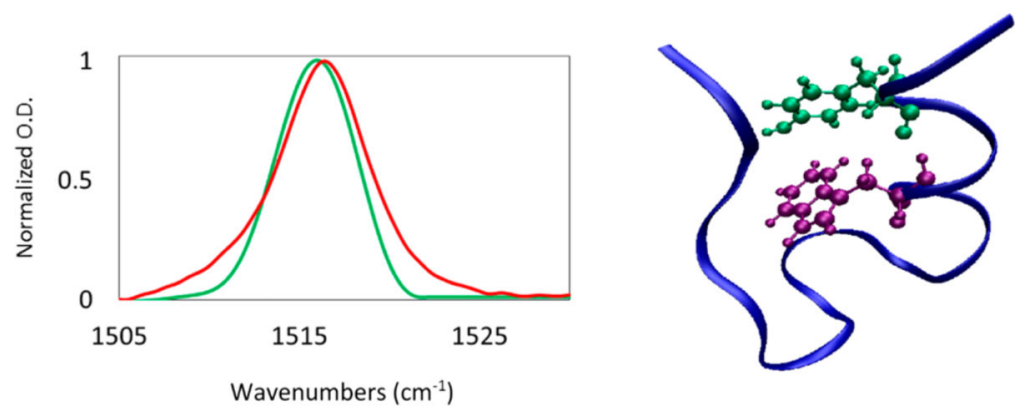

Figure 2.

(Left) Normalized FTIR spectra of Trp-cage miniprotein in native (red) and chemically denatured (green) states within the tyrosine ring mode region. (Right) Structure of Trp-cage highlighting hydrophobic core region (adapted from PDB 1L2Y). 

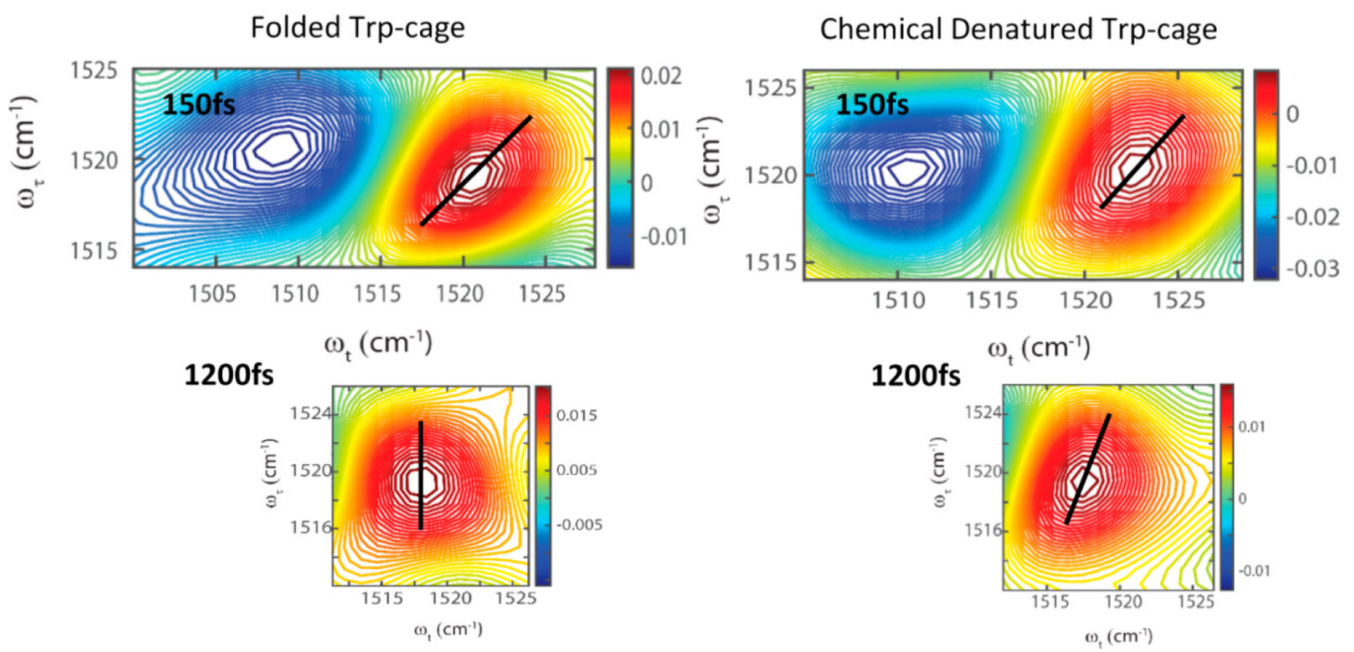

$1200 f s$

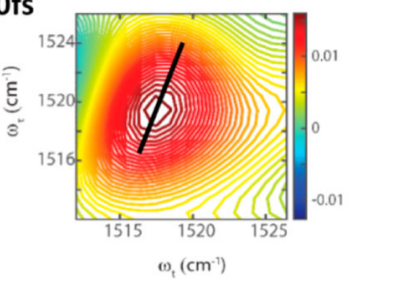

Figure 3.

2D IR spectra of Trp-cage miniprotein at different waiting times for the folded state (left) and denatured state (right), within the tyrosine ring mode region. 


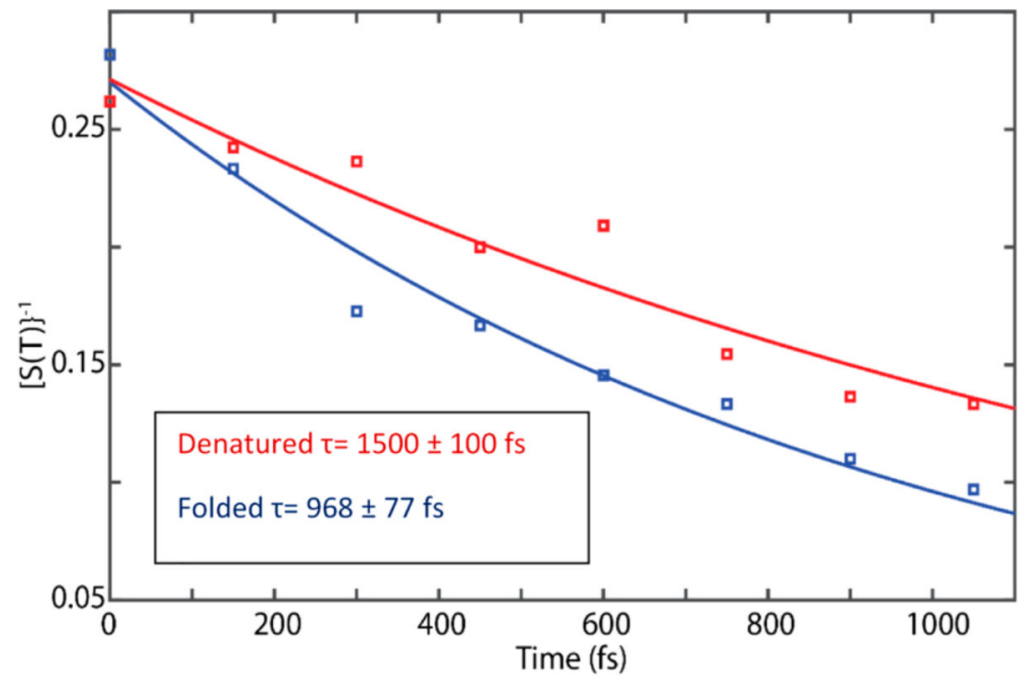

Figure 4.

Inverse slope $[S(T)]^{-1}$ versus waiting time of Trp-cage in folded (blue) and denatured states (red). 